\title{
Vision and lasers: An abstract
}

W. L. MAKOUS, DEPARTMENT OF PSYCHOLOGY UNIVERSITY OF WASHINGTON, Seattle, Wash. 98105 AND J. D. GOULD, IBM RESEARCH CENTER, Yorktown Heights, N. Y. 10598

This report is a summary of two, more comprehensive treatments of the effects of radiation emitted by lasers on the human visual system. Topics broached are: novel visual effects; threshold of damage to the eye, its mechanism, both theoretical and empirical estimates of it, and its relation to psychophysical brightness; computation of retinal irradiation by lasers; and a potential application of lasers.

Questions of both practical and theoretical interest arise in connection with the effects produced on the human visual system by the great density of energy and the great coherence (monochromaticity, uniformity of phase relationship, and collimation) available in the radiation from lasers. The purpose of this report is to summarize the parts of our more comprehensive treatments of the effects of lasers on the human visual system (Makous \& Gould, 1968; Gould \& Makous, in press), that might be especially relevant to readers of this journal.

The only truly novel visual effect caused exclusively by the high coherence of radiation available from lasers is the speckled appearance illumination by such radiation lends to a rough surface. Where this effect is undesirable, as in some visual displays, it can be eliminated by movement of the surface (e.g., rotation) or by insertion of a phase scrambler within the beam. Other novel effects are caused by a high concentration of energy within a small interval on one of the other dimensions of the radiation. For example, although lasers are not truly powerful devices, the energy stored within them can be concentrated into a sufficiently short period of time to generate gigawatts of power within that interval of time. Similarly, restricting the direction of propagation of the radiation to a single, highly collimated beam, permits the optical system of the eye to focus, by accident, almost the entire output of a laser onto a microscopic spot on the retina, covering only a few receptors. This obviously is dangerous to the eye.

A number of conclusions about these dangers can be made by means of a model that is restricted in applicability to the conditions holding at the threshold for damage to the eye. For example, at threshold, the damage is done by heat rather than by the electric field, electrostrictive stress, radiation pressure, or photochemical effects, for the energy in the radiation heats the tissue to damaging levels before any of these other variables approach damaging levels. Worst-case analysis of the model indicates that laser pulses containing less than 0.05 erg are certainly harmless; on the other hand, if a well-collimated pulse of 10 ergs, emitted from a ruby laser within $25 \mu \mathrm{sec}$, were to enter an emmetropic eye, it would be almost certain to cause some permanent damage (under worst-case conditions).

These theoretical estimates are consistent with empirically founded estimates, which are summarized in Fig. 1. A theoretical estimate slightly lower than the empirical estimate is to be expected because of a fundamental difference between the two approaches: the theoretical estimate defines conditions that certainly do not cause damage; the empirical estimates define, instead, a particular set of conditions that certainly do cause damage. In this particular case, the theoretical estimate is of greater general value.

The theoretical estimates of damage threshold are also consistent with psychophysical data. The upper limit of harmless laser pulses corresponds almost exactly to the energy that saturates the visual system, in the sense that further increases in energy cause no detectable increase in response of the visual system.

In this connection, the retinal irradiance that is due to a laser should not be computed without very careful consideration of each particular case. Of the formulas that have been given for this purpose, some err by as much as one million, and none are completely general.

One potential application of lasers is as a source of illumination for visual displays. Among the questions that arise here are the effects of the speckled appearance discussed above, and the consequences of the complete absence of screen persistence. A regeneration of the display at the rate of $60 \mathrm{~Hz}$, however, should avoid any undesirable consequences from this latter cause.

\section{REFERENCES}

GOULD, J. D. \& MAKOUS, W. L. Vision and lasers: Human factors of laser displays. Information Display, in press.

MAKOUS, W. L., \& GOULD, J. D. Vision and lasers: I-The effects of lasers on the human eye. IBM J. Res. Develop., 1968, 12, 257-271.

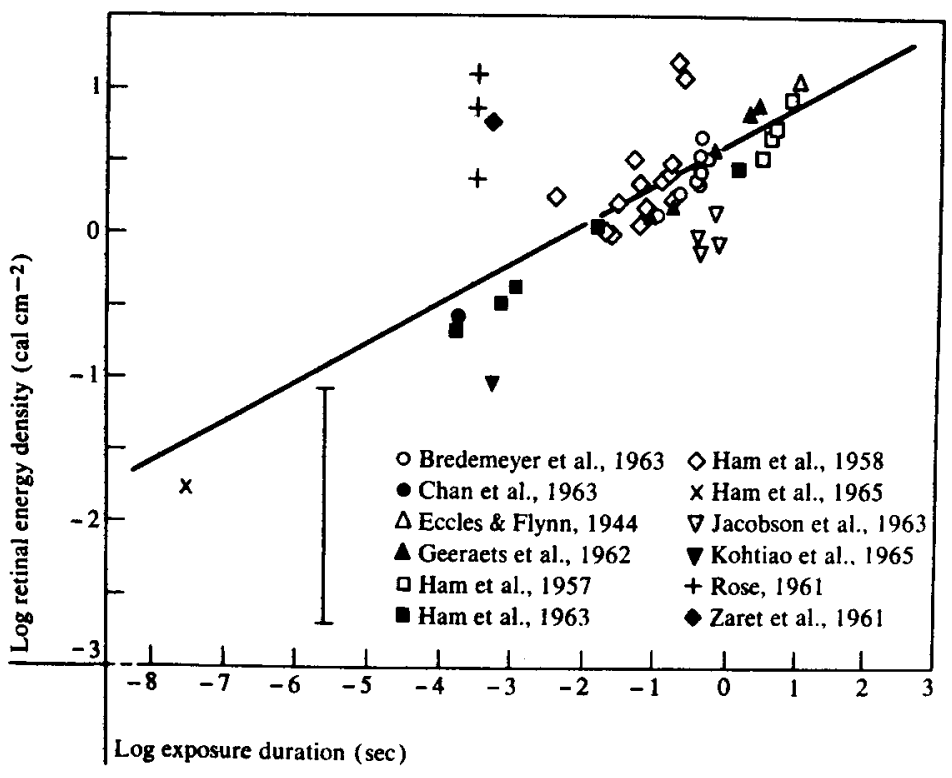

Fig. 1. Threshold for permanent damage to the retina by light irradiation of different durations. The brackets on the vertical line enclose the range within which the threshold must lie, in the worst case, according to theoretical estimate. Justification for the straight line, which was fitted by inspection, and the full references indicated in the key, are given in Makous \& Gould (in press). 